\title{
Epidemiologia, tratamento e profilaxia das infecções na leucemia linfóide crônica
}

\section{Epidemiology, treatment and prophylaxis of infections in chronic lymphocytic leukemia}

Márcia Garnical

Marcio Nucci $i^{2}$
Infecção é uma freqüente complicação em pacientes com leucemia linfocítica crônica (LLC). O risco de infecção é maior à medida que a doença avança, e guarda estreita correlação com a dosagem de imunoglobulinas no sangue. Embora o defeito imune primário na LLC seja deficiência de imunoglobulinas, as diversas modalidades de tratamento comprometem outros elementos do sistema imune, ampliando o espectro de agentes infecciosos. Este texto sumariza o conhecimento atual sobre os riscos de infecção com as diversas modalidades de tratamento e apresenta recomendações para o manejo destas infecções, seja na prevenção seja no tratamento. Tais recomendações são colocadas em graus de evidência tanto do ponto de vista de sua força quanto da qualidade da evidência. Rev. bras. hematol. hemoter. 2005;27(4):290-300.

Palavras-chave: Leucemia linfocítica crônica; infecção; tratamento; profilaxia.

\section{Introdução}

Leucemia linfocítica crônica (LLC) é a leucemia de maior freqüência entre adultos do mundo ocidental, sendo responsável por $30 \%$ de todas as leucemias nesta população. ${ }^{1}$ Infecções graves ocorrem em mais da metade dos casos, com uma incidência de 0,47 episódios de infecção (moderada a grave) por paciente/ano. ${ }^{2}$ Estima-se que até $50 \%$ dos pacientes com LLC sofrem de infecções recorrentes. ${ }^{3}$ Além disso, infecção é principal causa de óbito em pacientes com LLC. ${ }^{4} \mathrm{O}$ risco de infecção é maior com doença avançada e guarda estreita correlação com os níveis de imunoglobulinas no sangue. ${ }^{5}$

O comprometimento imunológico na LLC é multifatorial e ocorre em diversos momentos da evolução da doença, iniciando no diagnóstico e se estendendo durante todo o tratamento. A alteração mais característica é a hipogamaglobulinemia, presente em todo o curso da doença. ${ }^{3}$ A disfunção na imunidade humoral com acentuada hipogamaglobulinemia é somada a outras alterações relacionadas ao tratamento. Diferentes esquemas de poliquimioterapia, análogos da purina, corticosteróides, anticorpos monoclonais e o transplante de células progenitoras hematopoiéticas (TCPH) constituem o arsenal terapêutico na LLC e contribuem, cada um, para aumentar a imunodeficiência e o risco de infecção. Assim, à hipogamaglobulinemia somam-se outros defeitos na imunidade, incluindo graves alterações na imunidade mediada por linfócitos T e neutropenia. (Tabela 1)

\section{Risco de infecção}

Antes do tratamento

A principal imunodeficiência nos pacientes com LLC é a hipogamaglobulinemia, ocorrendo em 100\% dos pacientes. Embora a intensidade da deficiência seja variável em cada paciente, ela tende a piorar à medida que a doença

\footnotetext{
${ }^{I}$ Médica infectologista do Serviço de Hematologia e Unidade de Transplante de Medula Óssea, Hospital Universitário Clementino Fraga Filho, UFRJ. ${ }^{2}$ Prof. adjunto, Departamento de Clínica Médica, Serviço de Hematologia e Unidade de Transplante de Medula Óssea, Chefe do Laboratório de Micologia, Hospital Universitário Clementino Fraga Filho, UFRJ.
}

Serviço de Hematologia, Hospital Universitário Clementino Fraga Filho, Universidade Federal do Rio de Janeiro.

Correspondência para: Marcio Nucci

Hospital Universitário Clementino Fraga Filho

Av. Brigadeiro Trompovsky s/n

21941-590 - Rio de Janeiro-RJ - Brasil

Tel: +5521-25622463 Fax: +5521-25622460

E-mail: mnисci@hucf.ufrj.br 
Tabela 1

Risco de infecção em LLC

\begin{tabular}{|c|c|c|c|}
\hline $\begin{array}{l}\text { Fase da doença / } \\
\text { tratamento }\end{array}$ & Defeito na imunidade & Patógenos & Comentários \\
\hline Pré-Tratamento * & $\lg G++/ \lg A+$ & $\begin{array}{c}\text { Bactérias ++; Fungos +; } \\
\text { Vírus +/- }\end{array}$ & Neutropenia em casos avançados \\
\hline $\begin{array}{c}\text { Quimioterapia } \\
\text { convencional com agentes } \\
\text { alquilantes }\end{array}$ & $\begin{array}{c}\lg G_{++} / \lg A_{+} \\
\text {Neutropenia + }\end{array}$ & $\begin{array}{c}\text { Bactérias ++; Fungos ++; Vírus +; } \\
\text { Micobactérias - }\end{array}$ & $\begin{array}{l}\text { Ciclofosfamida + prednisona: maior } \\
\text { risco para pneumocistose }\end{array}$ \\
\hline Análogos da purina *** & $\begin{array}{l}\lg \mathrm{G}_{++} / \lg \mathrm{A}_{+} \\
\text {Neutropenia }+/++ \\
\text { Imunidade mediada por } \\
\text { linfócitos } \mathrm{T}+++\end{array}$ & $\begin{array}{c}\text { Bactérias (incluindo Listeria, Nocardia) } \\
+++; \text { Fungos }++; \text { Vírus }+++; \\
\text { Micobactérias }++{ }^{* *} \\
\text { Pneumocystis jiroveci }++\end{array}$ & $\begin{array}{l}\text { Risco aumentado com uso } \\
\text { concomitante de corticosteróides, } \\
\text { estágios avançados, disfunção renal, } \\
\text { regimes diferentes de quimioterapia } \\
\text { prévia e } C D 4<50 \text { células } / \mu \mathrm{L} \\
\text { Depressão na imunidade }>1 \text { ano após } \\
\text { suspensão }\end{array}$ \\
\hline Alemtuzumab & Linfopenia +++ & $\begin{array}{l}\text { Pneumocystis jiroveci +;Vírus ++; } \\
\text { Fungos (infecção superficial) + + }\end{array}$ & $\begin{array}{l}\text { Risco maior em pacientes refratários a } \\
\text { fludarabina ou a esquemas anteriores }\end{array}$ \\
\hline $\mathrm{TCPH}$ & $\begin{array}{l}\text { Autólogo: neutropenia ++ } \\
\text { Imunidade celular++ } \\
\text { Alogênico: } \\
\text { Neutropenia +++/- } \\
\text { Imunidade celular+++ } \\
\text { Imunidade humoral ++ }\end{array}$ & $\begin{array}{c}\text { Autólogo: } \\
\text { Bactérias ++; Vírus + } \\
\text { Alogênico: } \\
\text { Bacteriano } \\
\text { Infecção Fúngica Invasiva } \\
\text { Infecção Viral disseminada }\end{array}$ & $\begin{array}{c}\text { Presença e gravidade de doença do } \\
\text { enxerto contra o hospedeiro determina } \\
\text { o risco de infecção em TCPH } \\
\text { alogênico }\end{array}$ \\
\hline
\end{tabular}

progride. ${ }^{6}$ Essa alteração está presente em todo o curso da doença e, a despeito da melhora em diversos parâmetros, o tratamento da LLC não eleva os níveis de imunoglobulinas no sangue na maioria dos pacientes. ${ }^{7,8}$ A principal imunoglobulina afetada é a IgG, dos subtipos IgG3 e IgG4 e, com menor freqüência, IgA e IgM. ${ }^{1}$ Há uma relação estreita entre os níveis de IgG e a freqüência e gravidade de infecções, mas no caso de $\operatorname{IgA}$ e IgM esta correlação não é muito clara. ${ }^{1}$ Por outro lado, a freqüente ocorrência de infecções sinopulmonares é, em parte, atribuída à deficiência de IgA. Além da hipogamaglobulinemia, pacientes com LLC também podem apresentar alterações na ativação do sistema complemento, com uma resposta imune antibacteriana ineficaz. ${ }^{9}$ Estas alterações são mais intensas nas fases avançadas da doença, mas sua participação no risco de infecções não é muito clara, uma vez que não se demonstrou uma relação direta entre a falha de opsonização e um aumento na freqüência de infecções. ${ }^{10}$ Outro defeito na imunidade que pode estar presente ao diagnóstico é a neutropenia, mas este evento é raro.

Os locais mais comuns de infecção são os pulmões, seios paranasais, trato urinário e pele..$^{1,3,11} \mathrm{O}$ risco de adquirir infecção é tão maior quanto mais avançada for a doença e mais baixos forem os níveis de IgG no sangue..$^{2-4,7}$ Os patógenos mais freqüentes são os germes encapsulados (Streptococcus pneumonia, Haemophilus influenzae),
Staphylococcus aureus e bactérias entéricas Gram-negativas. Infecções fúngicas são raras, assim como infecções virais ou por micobactérias. ${ }^{5}$

\section{Durante o tratamento}

No decorrer da doença, o paciente é exposto a diversas modalidades de tratamento, incluindo agentes quimioterápicos alquilantes, corticosteróides, imunoterapias, TCPH e combinações destes agentes. Além do risco de infecção associado à hipogamaglobulinemia, outros defeitos na imunidade ocorrem, diferindo em cada estágio e em cada momento do tratamento. Entretanto, as diferentes modalidades de tratamento em geral acarretam riscos específicos, sendo possível definir um padrão de alterações imunológicas para cada fase e prever o tipo de infecção.

\section{a) Quimioterapia}

Clorambucil e ciclofosfamida são os quimioterápicos mais utilizados no tratamento inicial da LLC, com ou sem corticosteróides. Em geral, estes esquemas causam muito pouca neutropenia, e as alterações na imunidade mediada por linfócitos T dependem das doses de corticosteróides bem como do número de ciclos a que o paciente é exposto.

Esquemas poliquimioterápicos têm maior potencial de induzir neutropenia e, conseqüentemente, maior risco de infecção. Numa comparação randomizada entre clorambucil 
+ prednisona e ciclofosfamida + vincristina + prednisona (COP), houve cinco óbitos por infecção em 62 pacientes que receberam $\mathrm{COP}$ e apenas um em 60 pacientes que receberam clorambucil + prednisona. ${ }^{12}$ Em outro estudo, a exposição a mais de um regime de quimioterapia foi o principal fator de risco para infecção grave. ${ }^{13}$

b) Análogos da purina

A introdução da fludarabina, usada quer em primeira ou segunda linha no tratamento, resulta em uma profunda depressão na imunidade mediada por linfócitos T. ${ }^{14}$ Após três ciclos de fludarabina há uma significante diminuição na contagem de linfócitos T CD4 e CD8, persistindo por mais de um ano após a suspensão do tratamento. Quando administrada isoladamente, a fludarabina produz neutropenia em $15 \%$ a $75 \%$ dos pacientes. ${ }^{15}$ Quando usada em primeira linha, a fludarabina aumenta em 1,58 vezes o risco de infecção comparado com regimes de quimioterapia utilizando agentes alquilantes. ${ }^{16} \mathrm{Em}$ um estudo randomizado, o uso de fludarabina isoladamente ou em combinação com clorambucil resultou em maior freqüência de infecções, comparado com um grupo que recebeu clorambucil. ${ }^{17} \mathrm{~A}$ maior diferença entre os grupos ocorreu nas infecções virais, especialmente causadas por herpes vírus. Além disso, houve infecções por microorganismos pouco prevalentes em LLC, como micobactérias e Legionella. Esta mudança no espectro de infecções é uma das principais características do uso de fludarabina no tratamento da LLC. Tais infecções incluem: infecções bacterianas, como listeriose, nocardiose e legionelose; infecções por micobactérias, principalmente tuberculose; infecções fúngicas disseminadas ou superficiais por Candida sp., Aspergillus sp., Cryptococcus neoformans e Pneumocystis jiroveci; infecções virais por cytomegalovirus (CMV), varicella-zoster e herpes simplex; e infecções por parasitas, como criptosporidiose. ${ }^{18-24} \mathrm{O}$ risco de desenvolver estas infecções é maior em pacientes previamente tratados com outros esquemas, pacientes que recebem corticosteróides concomitantemente e pacientes que não respondem ao tratamento com fludarabina. ${ }^{18,25}$

Cladribina, outro análogo da purina, também é usado em monoterapia ou em combinação no tratamento da LLC, porém, o impacto dessa droga na imunidade celular é menor em relação à fludarabina. Neutropenia febril ocorre em cerca de $20 \%$ dos casos, e pneumonias, sinusite e herpes zoster são as infecções mais freqüentes. ${ }^{26}$

\section{c) Anticorpos monoclonais}

Recentemente, novas drogas foram incorporadas ao arsenal terapêutico da LLC, como rituximab (anti-CD20) e alemtuzumab (anti-CD52). Estes agentes são geralmente usados em associação com outras drogas, como fludarabina, em pacientes previamente tratados que apresentam progressão de doença. $\mathrm{O}$ rituximab causa significante redução na contagem de linfócitos B no sangue, com leve diminuição nos níveis de imunoglobulinas. Entretanto, tais alterações não resultam em aumento no risco de infecção. ${ }^{15}$ Por outro lado, o alemtuzumab produz prolongada redução na contagem de linfócitos B e linfócitos T CD4 e CD8, causando infecções associadas à depressão na imunidade mediada por linfócitos T, como pneumocistose, herpes zoster, citomegalovirose, infecções fúngicas invasivas e candidíase mucocutânea. ${ }^{27} \mathrm{~A}$ infecção mais frequientemente relatada é citomegalovirose. ${ }^{27-29} \mathrm{O}$ risco de infecção é maior em pacientes tratados previamente com múltiplos esquemas. ${ }^{27} \mathrm{Em}$ um estudo prospectivo, pacientes que responderam à terapia com fludarabina com ou sem ciclofosfamida foram randomizados a receber ou não alemtuzumab. Apesar do pequeno número de pacientes que receberam alemtuzumab, o estudo foi suspenso devido à ocorrência de infecções graves em sete de 11 pacientes (uma aspergilose invasiva, quatro citomegaloviroses, uma tuberculose pulmonar e uma herpes zoster). ${ }^{30}$

\section{d) Esplenectomia}

Alguns pacientes com LLC têm indicação de esplenectomia em situações como anemia hemolítica auto-imune, trombocitopenia auto-imune, esplenomegalia e citopenias causadas por hiperesplenismo. ${ }^{31}$ A cirurgia está associada a sepse no pós-operatório, com significante morbidade e mortalidade. ${ }^{32}$ Além dos riscos imediatos, a esplenectomia tende a acentuar a deficiência na imunidade humoral.

\section{e) $\mathrm{TCPH}$}

O TCPH tem sido utilizado no tratamento da LLC, particularmente em pacientes jovens com doença de alto risco. Em geral, o risco de infecção acompanha as diferentes modalidades de tratamento (transplante autólogo, alogênico mieloablativo e não-mieloablativo), não sendo muito diferente daquele relatado em pacientes com outras doenças. $\mathrm{O}$ transplante autólogo resulta em menor morbidade e mortalidade que o transplante alogênico, embora em uma comparação não randomizada de um único centro a mortalidade até o dia +100 não tenha sido diferente. ${ }^{33} \mathrm{O}$ transplante alogênico não-mieloablativo ou de intensidade reduzida tem sido utilizado mais recentemente na LLC, com menor toxicidade que o transplante mieloablativo. ${ }^{34}$ Após o TCPH alogênico, os pacientes apresentam risco de infecções fúngicas invasivas, infecções virais graves e infecções por micobactérias. ${ }^{35-39}$

\section{Manejo de infecções}

O manejo de problemas infecciosos em pacientes com LLC requer uma análise dos riscos de infecção baseado no tipo de tratamento prévio e atual a que o paciente foi submetido. A seguir, serão apresentadas recomendações para o manejo de infecções. Cada recomendação é classificada em 
Tabela 2

Categorização do nível de evidência

\begin{tabular}{cc}
\hline Categoria & Definição \\
\hline $\begin{array}{c}\text { Nível de } \\
\text { recomendação }\end{array}$ & \\
A & Forte evidência para recomendar \\
B & Moderada evidência para recomendar \\
C & Fraca evidência para recomendar \\
D & Moderada evidência para contra indicar \\
E & Forte evidência para contra-indicar \\
\hline $\begin{array}{c}\text { Qualidade da } \\
\text { evidência }\end{array}$ & $\begin{array}{c}\text { Evidência de } \geq 1 \text { estudo randomizado, controlado } \\
\text { I }\end{array}$ \\
II & $\begin{array}{c}\text { Evidência de } \geq 1 \text { ensaio clinico não randomizado, } \\
\text { ou de estudo de coorte ou caso controle, ou } \\
\text { estudos não controlados com } \\
\text { resultados dramáticos }\end{array}$ \\
III & $\begin{array}{c}\text { Evidência de autoridades, série de casos ou de } \\
\text { experiência clínica de "experts" }\end{array}$ \\
\hline
\end{tabular}

nível de evidência, conforme a tabela 2. Como na maioria das situações não há estudos randomizados avaliando diferentes estratégias de manejo de infecções em pacientes com LLC, as recomendações que se seguem são baseadas na observação de uma maior frequiência de determinadas infecções em grupos de maior risco e, quando disponíveis, dados de estudos em outras populações de pacientes. Por esta razão, sempre que for o caso, serão apresentados dois níveis de evidência, sendo o primeiro aquele relativo à indicação geral (em outras populações de pacientes) e o outro à LLC. Quando apenas um nível de evidência for apresentado, o mesmo se refere à LLC. As recomendações apresentadas serão divididas em: abordagem inicial, estratégias preventivas (incluindo medidas profiláticas, preemptivas e vacinação) e terapêuticas (Tabela 3).

\section{Abordagem inicial}

Na avaliação inicial de pacientes com LLC, recomenda-se dosar as imunoglobulinas no sangue [AII], tanto para avaliar a necessidade de profilaxia para pacientes com níveis baixos de $\operatorname{IgG}(<600 \mathrm{mg} / \mathrm{dL})$, como para comparação com avaliações futuras. Em pacientes que recebem fludarabina ou alemtuzumab, recomenda-se fazer a contagem de linfócitos CD4 periodicamente, pois algumas medidas preventivas são recomendadas para pacientes com contagens abaixo de 50 células/ $\mu \mathrm{L}$ [BII]. Outras avaliações, tais como contagem global e específica de leucócitos, dosagem da proteína $\mathrm{C}$ reativa no sangue, exames radiológicos e de culturas, seguem as condutas gerais para infecção, aplicáveis a qualquer paciente.

\section{Estratégias preventivas}

Com o intuito de reduzir ou minimizar a ocorrência de eventos infecciosos em pacientes com LLC, estratégias preventivas podem ser tomadas baseando-se nos tipos e graus de imunodeficiência presentes em cada época do curso da doença. Estas estratégias podem ser resumidas em quatro grupos: profilaxia, uso de fatores estimuladores de crescimento de colônias, reposição de imunoglobulinas e vacinação.

\section{a) Profilaxia}

Não há estudos randomizados testando diferentes agentes na profilaxia de infecções em LLC. Pacientes que recebem análogos das purinas (especialmente em combinação com corticosteróides) ou alemtuzumab e que apresentem outros fatores de risco para infecção (doença em estágios avançados, tratamento prévio, mucosite grave ou neutropenia) podem se beneficiar de profilaxia antimicrobiana [BIII]. Anaissie e colaboradores propuseram um algoritmo para profilaxia em pacientes recebendo fludarabina, que leva em conta a presença de fatores de risco. ${ }^{18}$ Os pacientes de maior risco de infecção são aqueles: a) com estágios Rai III ou IV; b) que tenham recebido vários regimes de quimioterapia; c) que receberam ou estão recebendo corticosteróides; d) que apresentam contagem de CD4 $<50 / \mu \mathrm{L}$; e) que apresentem disfunção renal. Em pacientes com vários fatores de risco, os autores recomendam o uso de sulfametoxazol-trimetoprim (SMZ-TMP, um comprimido contendo $800 \mathrm{mg}$ de sulfametoxazol duas vezes ao dia). Tal estratégia previne infecção por Pneumocystis jiroveci [AI; AIII], Listeria, Legionella, Nocardia e outras bactérias mais comuns (particularmente as bactérias encapsuladas), e pode ser estendida a pacientes em uso de alemtuzumab [BIII]. O esquema intermitente de três dias consecutivos na semana se mostrou eficaz para prevenir pneumocistose em crianças com leucemia linfóide aguda, mas sua eficácia em pacientes com LLC e em outras infecções não está estabelecida [AI; CIII.$^{40} \mathrm{~A}$ duração da profilaxia também não está estabelecida, mas deve-se considerar o uso prolongado [BIII], uma vez que as contagens de CD4 permanecem baixas por até um ano depois do uso de fludarabina. ${ }^{14}$

Além da profilaxia com SMZ-TMP, os pacientes deverão ser acompanhados para identificação precoce de doenças virais (herpes simplex, herpes zoster e, em pacientes recebendo alemtuzumab, citomegalovirose), principalmente se a contagem de linfócitos T CD4 estiver baixa. Para a identificação precoce de herpes simplex e herpes zoster, a orientação ao paciente quanto ao aparecimento de manifestações clínicas pode ser útil [CIII]. Alguns autores sugerem que pacientes que apresentem alguma lesão cutânea sugestiva destas infecções tomem uma dose de aciclovir (400 mg VO) ou valaciclovir (1.000 mg) [CIII]. Para a citomegalovirose, a melhor estratégia é a realização periódica de antigenemia e terapia preemptiva com ganciclovir $(5 \mathrm{mg} / \mathrm{kg} 12 / 12 \mathrm{~h}$ por duas semanas) [AI; BIII], ${ }^{28,41}$ foscarnet $(60 \mathrm{mg} / \mathrm{kg} 12 / 12 \mathrm{~h}$ por duas semanas) [AI; BIII] ${ }^{42}$ ou valganciclovir $(900 \mathrm{mg}$ por dia) $[\mathrm{CIII}] .{ }^{28,43}$ 
Tabela 3

Recomendações para manejo de infecções em pacientes com leucemia linfocítica crônica

\begin{tabular}{|c|c|c|c|}
\hline Situação & Recomendação & Evidência & Comentários \\
\hline Avaliação inicial & Dosar imunoglobulinas no sangue & All & Níveis $<600 \mathrm{mg} / \mathrm{dL}$ associados com maior risco de infecção \\
\hline Avaliação inicial & Contagem de linfócitos CD4 no sangue & Bll & Pacientes que vão receber fludarabina e/ou alemtuzumab \\
\hline $\begin{array}{l}\text { Uso de fludarabina ou alemtuzumab + } \\
\text { fatores de risco* }\end{array}$ & $\begin{array}{c}\text { SMZ-TMP: } 800 \text { mg de SMZ 12/12 h VO } \\
\text { diariamente }\end{array}$ & Al; Alll & $\begin{array}{c}\text { Previne pneumocistose, listeriose, legionelose, nocardiose e } \\
\text { infecções bacterianas }\end{array}$ \\
\hline $\begin{array}{l}\text { Lesões cutâneas sugestivas de herpes } \\
\text { simplex ou herpes zoster durante } \\
\text { tratamento com fludarabina, } \\
\text { alemtuzumab e / ou corticosteróides }\end{array}$ & $\begin{array}{l}\text { Tomar uma dose de aciclovir ( } 800 \mathrm{mg}) \\
\text { ou valaciclovir }(1000 \mathrm{mg}) \mathrm{VO}\end{array}$ & CIII & Recomendação aos pacientes, antes de chegarem ao hospital \\
\hline $\begin{array}{l}\text { Uso de fludarabina ou alemtuzumab + } \\
\text { fatores de risco* }\end{array}$ & $\begin{array}{l}\text { Antigenemia para CMV e terapia } \\
\text { preemptiva caso positiva }\end{array}$ & $\mathrm{Al} ; \mathrm{BIII}$ & $\begin{array}{l}\text { Ganciclovir: } 5 \mathrm{mg} / \mathrm{kg} / \mathrm{d} \text { x } 14 \mathrm{~d} \text { IV [Al; BIII] } \\
\text { Foscarnet: } 60 \mathrm{mg} / \mathrm{kg} / \mathrm{d} \text { x } 14 \mathrm{~d} \text { IV [Al; BIII] } \\
\text { Valganciclovir: } 900 \mathrm{mg} / \mathrm{d} \text { x } 14 \mathrm{~d} \text { IV [CIII] }\end{array}$ \\
\hline Contagem de CD4 $<50 \mu \mathrm{L}$ & $\begin{array}{l}\text { Aciclovir: } 400 \mathrm{mg} \text { VO } 2 \text { a } 3 \text { x/d } \\
\text { Valaciclovir: } 500 \mathrm{mg} 8 / 8 \mathrm{~h} \text { VO }\end{array}$ & CIII & Manter até CD4>250 células/ $\mu \mathrm{L}[\mathrm{CIII}]$ \\
\hline $\begin{array}{l}\text { Uso de fludarabina ou alemtuzumab e } \\
\text { história de tuberculose }\end{array}$ & Isoniazida: $300 \mathrm{mg} / \mathrm{d}$ VO & $\mathrm{BI} ; \mathrm{ClII}$ & \\
\hline $\begin{array}{l}\text { Profilaxia primária de infecções } \\
\text { bacterianas }\end{array}$ & SMZTMP & DIII & Não está recomendado \\
\hline $\begin{array}{l}\text { Profilaxia secundária de infecções } \\
\text { bacterianas }\end{array}$ & $\begin{array}{c}\text { SMZTMP: } 800 \text { mg de SMZ 12/12 h VO } \\
\text { diariamente }\end{array}$ & BIII & $\begin{array}{l}\text { Para pacientes que apresentaram } \\
\text { infecção bacteriana grave }\end{array}$ \\
\hline Febre + calafrios & $\begin{array}{l}\text { Tomar } 1 \text { comprimido de } \\
\text { amoxacilina/clavulanato }\end{array}$ & Clll & $\begin{array}{c}\text { Recomendação aos pacientes antes de chegarem ao hospital. } \\
\text { Outras opções: quinolonas respiratórias, ampicilina/sulbactam, } \\
\text { cefuroxima }\end{array}$ \\
\hline Uso de alemtuzumab & Profilaxia antifúngica & DIl; DIII & Não recomendado \\
\hline $\mathrm{TCPH}$ & Aciclovir: $250 \mathrm{mg} / \mathrm{m} 2$ 8/8 h IV & $\mathrm{Al}$ & $\begin{array}{l}\text { Do condicionamento até a } \\
\text { recuperação medular }\end{array}$ \\
\hline TCPH alogênico & Fluconazol: $400 \mathrm{mg} / \mathrm{d}$ VO ou IV & $\mathrm{Al}$ & $\begin{array}{l}\text { Do condicionamento até a } \\
\text { recuperação medular }\end{array}$ \\
\hline TCPH autólogo & Fluconazol & DIII & $\begin{array}{l}\text { Não recomendado. Usar se expectativa de neutropenia } \\
\text { prolongada, mucosite grave ou uso recente de fludarabina [BIII] }\end{array}$ \\
\hline TCPH alogênico & Antigenemia semanal para CMV & Alll & Da pega até $D+100$ \\
\hline $\begin{array}{l}\text { TCPH alogênico com } \\
\text { antigenemia positiva para CMV }\end{array}$ & $\begin{array}{c}\text { Ganciclovir: } 5 \text { mg/kg } 12 / 12 \text { h IV x } 2 \\
\text { semanas }\end{array}$ & BIII & \\
\hline TCPH autólogo & SMZ-TMP: $800 \mathrm{mg}$ de SMZ 2x/d VO & BIII & Até 1 ano, especialmente após uso de fludarabina \\
\hline TCPH durante a fase de neutropenia & Profilaxia antibacteriana & DIII & Não recomendado \\
\hline TCPH alogênico com GVHD crônico & $\begin{array}{l}\text { Antibióticos ativos contra bactérias } \\
\text { encapsuladas }\end{array}$ & BIII & A escolha do agente depende da epidemiologia local \\
\hline $\begin{array}{l}\text { Profilaxia primária em neutropenia com } \\
\text { expectativa de neutropenia febril }=40 \%\end{array}$ & G-CSF: $5 \mu \mathrm{g} / \mathrm{kg} / \mathrm{d}$ & $\mathrm{Al} ; \mathrm{ClII}$ & $\begin{array}{c}\text { Não é recomendado em quimioterapia com expectativa de } \\
\text { neutropenia febril }<40 \%\end{array}$ \\
\hline Profilaxia secundária em neutropenia & G-CSF: $5 \mu \mathrm{g} / \mathrm{kg} / \mathrm{d}$ & BIII; CIII & $\begin{array}{l}\text { Para pacientes que apresentaram neutropenia febril em ciclo } \\
\text { precedente }\end{array}$ \\
\hline Neutropenia afebril & G-CSF: $5 \mu \mathrm{g} / \mathrm{kg} / \mathrm{d}$ & DII; DIII & Não é recomendado \\
\hline Neutropenia febril & G-CSF: $5 \mu \mathrm{g} / \mathrm{kg} / \mathrm{d}$ & DI; DIII & Não é recomendado rotineiramente \\
\hline $\begin{array}{l}\text { Hipogamaglobulinemia e / ou } \\
\text { história de infecção }\end{array}$ & $\begin{array}{l}\text { Imunoglobulina IV: } 400 \mathrm{mg} / \mathrm{kg} \\
3 / 3 \text { semanas }\end{array}$ & $\mathrm{Al}$ & $\begin{array}{l}\text { Embora evidência forte, o benefício é pequeno em confronto } \\
\text { com o alto custo }\end{array}$ \\
\hline
\end{tabular}


Tabela 3 (continuação)

Recomendações para manejo de infecções em pacientes com leucemia linfocítica crônica

\begin{tabular}{|c|c|c|c|}
\hline Situação & Recomendação & Evidência & Comentários \\
\hline Antes do tratamento & $\begin{array}{l}\text { Vacinas conjugadas para pneumococo, } \\
\text { Haemophilus influenzae, Influenza }\end{array}$ & CIII & Pouca resposta à vacina \\
\hline Febre + sintomas respiratórios & $\begin{array}{l}\text { Antibioticoterapia empírica contra } \\
\text { germes encapsulados }\end{array}$ & $\mathrm{B} \|$ & $\begin{array}{l}\text { Opções: cefuroxima, penicilinas com inibidores de } \\
\text { betalactamase, quinolonas respiratórias e macrolídeos }\end{array}$ \\
\hline $\begin{array}{l}\text { Neutropenia febril de curta duração em } \\
\text { pacientes de baixo risco }\end{array}$ & Quinolona respiratória & $\mathrm{Al} ; \mathrm{B} \|$ & $\begin{array}{l}\text { Serve para pacientes sem foco de infecção, calafrios ou } \\
\text { hipotensão (ver detalhes no texto) }\end{array}$ \\
\hline Neutropenia febril & Monoterapia empírica & $\mathrm{Al} ; \mathrm{BII}$ & \\
\hline $\begin{array}{l}\text { Febre + úlceras na mucosa oral, face ou } \\
\text { região perineal em pacientes } \\
\text { recebendo fludarabina }\end{array}$ & $\begin{array}{c}\text { Valaciclovir: } 1000 \mathrm{mg}^{2} \mathrm{x} / \mathrm{d} \text { VO } \\
\text { Aciclovir: } 250 \mathrm{mg} / \mathrm{m}^{2} 8 / 8 \mathrm{~h} \text { IV ou } \\
400 \mathrm{mg} \mathrm{5x/d} \mathrm{VO}\end{array}$ & $\begin{array}{l}\mathrm{Al} ; \mathrm{CIII} \\
\mathrm{Al} ; \mathrm{Clll}\end{array}$ & Suspeita de infecção por Herpes simples \\
\hline $\begin{array}{l}\text { Febre + sinais neurológicos em } \\
\text { pacientes recebendo fludarabina }\end{array}$ & $\begin{array}{l}\text { SMZTMP ou ampicilina + cefepima } \\
\text { TC e /ou RNM de crânio }\end{array}$ & $\begin{array}{l}\text { CIII } \\
\text { CIII }\end{array}$ & $\begin{array}{l}\text { Suspeita de meningite por Listeria } \\
\text { Suspeita de criptococose ou toxoplasmose }\end{array}$ \\
\hline $\begin{array}{l}\text { Febre + dispnéia em pacientes } \\
\text { recebendo fludarabina }\end{array}$ & SMZTMP em doses altas & BIII & Suspeita de pneumocistose \\
\hline $\begin{array}{l}\text { Febre de origem obscura em pacientes } \\
\text { recebendo alemtuzumab }\end{array}$ & $\begin{array}{l}\text { Antigenemia para CMV e terapia } \\
\text { preemptiva (ver drogas e doses acima) }\end{array}$ & BIII & Alto risco de reativação de CMV \\
\hline
\end{tabular}

* fatores de risco: com estágios avançados, vários regimes de quimioterapia, uso de corticosteróides, contagem de CD4 <50/ $\mu \mathrm{L}$, disfunção renal; SMZ/TMP: sulfametoxazol-trimetoprim; VO: via oral; IV: intravenoso CMV: cytomegalovirus; TCPH: transplante de células progenitoras hematopoiéticas;

Alguns estudos sugerem o uso de profilaxia contra Herpes simplex ou Varicella-zoster com aciclovir (400 mg duas a três vezes ao dia), valaciclovir (500 mg 8/8h) ou famciclovir $(500 \mathrm{mg} \mathrm{8/8} \mathrm{h}$ ) para pacientes com CD4 < 50 células $/ \mu \mathrm{L}$, continuando até que a contagem de linfócitos CD4 supere 250 células/ $\mu \mathrm{L}[\mathrm{CIII}],{ }^{28,44}$ mas o benefício deve ser pesado em confronto com o risco de resistência, toxicidade e custo. Pacientes com história prévia de tuberculose e uso de fludarabina ou alemtuzumab deverão ser avaliados quanto à indicação de quimioprofilaxia com isoniazida $(300 \mathrm{mg} / \mathrm{d})$ [BI; CIII] ${ }^{45-48}$ mas a duração da profilaxia não está estabelecida.

O uso rotineiro de profilaxia antibacteriana não está recomendado [DIII]. Entretanto, o uso diário de SMZ-TMP pode beneficiar pacientes que apresentaram uma infecção bacteriana grave (profilaxia secundária) [BIII]. ${ }^{49} \mathrm{~A}$ utilização de outros agentes para este fim não foi avaliada. Outra estratégia recomendada por alguns autores é a utilização preemptiva de antibacterianos. Segundo esta recomendação, os autores sugerem que pacientes com LLC que apresentam febre e calafrios tomem imediatamente um comprimido de amoxacilina com ácido clavulânico para tratar precocemente uma bacteremia por bactérias encapsuladas (especialmente pneumococo) [CIII] ${ }^{49}$ Outras opções válidas para esta terapia preemptiva são as quinolonas com atividade contra patógenos de infecções respiratórias comunitárias (quinolonas respiratórias - gatifloxacina, moxifloxacina e levofloxacina), ampicilina com sulbactam e cefuroxima.
O uso de alemtuzumab resulta em maior frequiência de candidíase mucosa, mas o uso profilático com fluconazol não é recomendado rotineiramente devido ao risco de seleção de espécies resistentes [DII; DIII]. ${ }^{28}$

Para pacientes que serão submetidos a TCPH, seguemse as recomendações dos TCPH para outras neoplasias hematológicas. ${ }^{50}$ Assim, os níveis de evidência apresentados neste parágrafo se referem a recomendações gerais para TCPH: a) profilaxia para herpes simplex com aciclovir $\left(250 \mathrm{mg} / \mathrm{m}^{2} 8 / 8 \mathrm{~h} \mathrm{IV}\right)$ até a recuperação medular [AI]; b) profilaxia antifúngica com fluconazol $(400 \mathrm{mg} / \mathrm{d})$ durante a fase de neutropenia para TCPH alogênico [AI]; para o TCPH autólogo a profilaxia antifúngica não é recomendada rotineiramente [DIII], a não ser em pacientes com perspectiva de longa duração de neutropenia (manipulação in vitro) e lesão grave de mucosa, ou aqueles que receberam recentemente fludarabina ou cladribina [BIII]; c) antigenemia semanal para CMV desde a pega do enxerto até o dia +100 [AIII] e ganciclovir $(10 \mathrm{mg} / \mathrm{kg} / \mathrm{d}$ por duas semanas) em caso de antigenemia positiva [BIII]; d) SMZ-TMP (800 mg duas vezes ao dia) para TCPH alogênico até seis meses após o transplante [AII], ou seis meses após suspensão de terapia imunossupressora [AI]; SMZ-TMP (mesma dose) para TCPH autólogo até um ano, especialmente após o uso de fludarabina [BIII]; e) não é recomendada a administração de profilaxia antibacteriana durante a fase de neutropenia [DIII], mas esta questão está sendo revista, uma vez que uma recente metanálise mostrou que o uso de quinolonas resultou em redução na mortalidade por infec- 
ção ${ }^{51}$; f) antibioticoterapia profilática contra bactérias encapsuladas para pacientes com doença do enxerto contra o hospedeiro crônica [BIII] (a escolha do agente depende da epidemiologia local).

b) Uso de fatores estimuladores de crescimento de colônias

O fator estimulador de colônias de granulócitos G-CSF tem sido empregado em pacientes que recebem terapia combinada de fludarabina com outros agentes, numa tentativa de manter a duração dos ciclos de quimioterapia e reduzir o período de neutropenia. ${ }^{29,52}$ Entretanto, a relação custo-benefício desta medida é bastante questionável. Guias práticos para o uso de G-CSF foram publicadas e indicam que para a profilaxia primária (uso de G-CSF antes do primeiro ciclo de quimioterapia), o G-CSF só tem boa relação custo-benefício quando o risco de neutropenia febril for $\geq 40 \%$ [AI; $\mathrm{CIII}] .^{53}$ Para a profilaxia secundária (uso do G-CSF depois do primeiro ciclo de quimioterapia), recomenda-se o uso do G-CSF para pacientes que apresentaram neutropenia febril e que irão receber um novo ciclo de quimioterapia igual [BIII; $\mathrm{CIII}]{ }^{53} \mathrm{O}$ uso de G-CSF para pacientes já neutropênicos e afebris não está indicado [DII; DIII], ${ }^{53}$ assim como o seu uso rotineiro em pacientes neutropênicos febris [DI; DIII] ${ }^{53}$

\section{c) Imunoglobulina}

O uso de imunoglobulina IV está associado com uma redução na freqüência de infecções em pacientes com LLC [AI]..$^{54-58}$ No maior estudo, 84 pacientes com hipogamaglobulinemia e/ou história de infecção foram randomizados a receber imunoglobulina IV $(400 \mathrm{mg} / \mathrm{kg}$ a cada três semanas) ou placebo. O uso de imunoglobulina resultou em menor frequiência de episódios de infecção bacteriana, mas não houve diferenças nas freqüências de infecções causadas por outros agentes. ${ }^{54}$ Uma dose menor de imunoglobulina foi testada em outro estudo, mas o número de pacientes era pequeno para detectar diferenças relevantes. ${ }^{58} \mathrm{~A}$ principal limitação do uso de imunoglobulinas IV é o seu alto custo. Desta forma, levando em considerando este aspecto, e o fato de que a prevenção pode ser obtida com o uso diário de SMZTMP (ver acima), a reposição intravenosa de imunoglobulina não é recomendada rotineiramente [CI]. O seu emprego em populações selecionadas (pacientes com hipogamaglobulinemia e repetidos episódios de infecção sino-pulmonar grave) pode ser considerado [CIII].

\section{d) Imunização}

Infecções respiratórias causadas por vírus, incluindo Influenza, causam grande morbidade em pacientes idosos, e a vacinação é uma medida preventiva eficaz. Entretanto, pacientes com LLC apresentam resposta muito ruim a esta e a outras vacinas, tais como anti-pneumococo e antiHaemophilus influenzae. Por outro lado, o uso de vacinas conjugadas pode melhorar a resposta e oferecer proteção contra bactérias encapsuladas [CIII]..$^{59,60}$ Assim, não há recomendação de vacinação especial para pacientes com LLC. Entretanto, se o seu uso for considerado, deve-se administrar a vacina antes do início do tratamento [CIII] ${ }^{49}$

\section{Tratamento}

As medidas terapêuticas deverão ser norteadas primariamente pelo tipo de tratamento em uso e pela imunossupressão prévia. Pacientes refratários ou com doenças de estádio avançado apresentam risco infeccioso acentuado, merecendo terapias antimicrobianas mais intensas e geralmente em ambiente hospitalar. Existe pouca recomendação na literatura especificando o tratamento para infecções na LLC. A seguir, estão recomendações baseadas no tipo de tratamento:

a) pacientes sem tratamento prévio ou após quimioterapia com clorambucil e prednisona, ou outro esquema menos intenso

Todo paciente com LLC que apresente febre deve ser considerado como tendo infecção até prova contrária. $\mathrm{O}$ quadro infeccioso deverá ser investigado principalmente quanto à presença de infecções do trato respiratório, com exame de imagem de tórax e seios da face, bem como contagem de leucócitos no sangue, para avaliar se o paciente se encontra neutropênico [BIII]. O esquema antimicrobiano no paciente virgem de tratamento, que não apresenta neutropenia, deve ser guiado pela avaliação inicial. Em caso de sintomas respiratórios, deve-se iniciar antibioticoterapia empírica para germes encapsulados (cefuroxima, penicilinas semi-sintéticas com inibidores de betalactamase, quinolonas respiratórias ou macrolídeos) [BII]. ${ }^{49}$ A freqüência de neutropenia é tão maior quanto mais intenso for o esquema de quimioterapia. Além das recomendações acima descritas para germes encapsulados, a conduta muda se o paciente tem neutropenia. O uso de antibioticoterapia empírica oral está recomendado se o paciente tem expectativa de neutropenia de curta duração $(<7$ dias), desde que não apresente algum foco de infecção bacteriana, ou sinais e sintomas sugestivos de infecção grave (calafrios, hipotensão) [AI; BII] ${ }^{61}$ As características que favorecem a classificação da neutropenia como sendo de "baixo risco" são: contagem absoluta de neutrófilos ou monócitos $\geq 100 / \mathrm{mm}^{3}$, radiografia de tórax normal, testes de função hepática ou renal minimamente alterados, expectativa de duração da neutropenia $<7$ dias ou de resolução da mesma < 10 dias, ausência de cateter venoso central, e ausência de alterações neurológicas mentais, dor abdominal, vômito ou diarréia. Embora para outras populações de pacientes qualquer quinolona possa ser usada, em pacientes com LLC as quinolonas respiratórias devem ser as escolhidas, porque também oferecem proteção contra o pneumococo. Em situações de neutropenia febril que não se enquadram nos critérios acima, devem-se adminis- 
trar antibióticos por via sistêmica, em geral monoterapia com uma cefalosporina de $4^{a}$ geração, carbapenem ou piperacilina-tazobactam $[\mathrm{AI} ; \mathrm{BII}] .{ }^{61}$

b) Após o uso de análogos da purina

$\mathrm{Na}$ avaliação de pacientes recebendo fludarabina, devem-se considerar os determinantes de maior risco de infecção: estágio (maior risco com doença avançada), tratamento prévio (maior risco quanto mais regimes de quimioterapia prévia e ciclos de fludarabina), uso concomitante de corticosteróides (maior risco), função renal (maior risco com disfunção do órgão) e contagem de linfócitos CD4 (risco aumentado com contagens $<50$ células $/ \mu \mathrm{L}$ ). A avaliação inicial inclui história, exame físico, leucometria com contagem diferencial, exames de função hepática e renal, hemoculturas, culturas de outros sítios (de acordo com os sinais clínicos), e antigenemia para CMV [BIII] ${ }^{18}$ Outros exames incluem culturas, sorologias e exames de imagem para afastar infecção por micobactérias, $P$. jiroveci e fungos (particularmente candidíase mucosa e criptococose) [CIII]. Em seguida deve-se iniciar terapia empírica guiada para o sítio de infecção identificado, ou terapia ampla, se o paciente estiver neutropênico. As mesmas recomendações descritas no item anterior para antibioticoterapia em pacientes neutropênicos se aplicam aos pacientes em uso de fludarabina. Para pacientes com manifestações clínicas localizadas em sítios específicos, as seguintes recomendações devem ser seguidas: presença de úlceras na mucosa oral, face, região perineal (sugestivas de herpes simplex): acrescentar valaciclovir $(1.000 \mathrm{mg} 2 \mathrm{x} / \mathrm{d} \mathrm{VO})[\mathrm{AI} ; \mathrm{CIII}]^{62}$ ou aciclovir $\left(250 \mathrm{mg} / \mathrm{m}^{2} 8 / 8 \mathrm{~h}\right.$ IV ou $\left.400 \mathrm{mg} 5 \mathrm{x} / \mathrm{d} \mathrm{VO}\right)$ [AI; $\mathrm{CIII}^{63,64}$; sinais neurológicos (suspeita de listeriose): SMZTMP ou ampicilina + cefepima [CIII], tomografia computadorizada de crânio, ressonância nuclear magnética, sorologia para Cryptococcus neoformans e Toxoplasma gondii; sinais de pneumonia (suspeita de pneumocistose): SMZTMP em doses altas (particularmente se o paciente recebeu quimioterapia prévia e/ou corticosteróides concomitantemente à fludarabina), exame de escarro ou lavado broncoalveolar para bactérias, fungos, vírus, micobactérias e $P$ jiroveci $[\mathrm{BIII}] .{ }^{18}$

c) Após o uso de anticorpos monoclonais

Pacientes recebendo alemtuzumab devem ser conduzidos de forma similar à recomendada para pacientes que recebem fludarabina. Além disso, uma atenção especial deve ser dada à reativação de CMV. Além da monitorização de antigenemia (ou PCR), como foi discutido no item de profilaxia, estes testes devem ser feitos no caso de febre, tanto na investigação inicial de febre sem sinais clínicos evidentes de infecção, quanto em pacientes persistentemente febris, incluindo pacientes neutropênicos [BIII].$^{28}$ No caso de antigenemia positiva, é recomendado um curso de ganciclovir, seguindo as mesmas recomendações do item "profilaxia". Se a antigenemia para CMV não for disponível, deve-se assumir que uma febre de origem obscura seja devida a reativação de cytomegalovirus, e iniciado ganciclovir $[\mathrm{CIII}] .{ }^{28}$

\section{d) Após TCPH}

O manejo de infecções no paciente com LLC que é submetido a TCPH não difere substancialmente do manejo de infecções em outras doenças de base. Uma revisão das recomendações do manejo de infecções em pacientes submetidos a TCPH foge do escopo deste artigo e pode ser encontrada em publicações recentes. ${ }^{65-68}$

\section{Sumário das recomendações}

A seguir serão apresentadas as recomendações para manejo de infecções no paciente com LLC.

1. Avaliação inicial do paciente

- Dosar imunoglobulinas no sangue.

- Se o paciente for receber fludarabina e/ou alemtuzumab, contar linfócitos CD4 no sangue.

- Educar o paciente quanto à presença de febre e calafrios, e orientá-lo a tomar imediatamente uma dose de antibiótico antipneumocócico (amoxacilina/clavulanato ou outro agente) caso apresente febre e calafrios.

- Educar o paciente quanto ao surgimento de lesões cutâneas vesiculares sugestivas de herpes simplex ou herpes zoster e orientá-lo a tomar imediatamente uma dose de $400 \mathrm{mg}$ de aciclovir.

\section{Profilaxias}

a) pacientes sem fludarabina e / ou alemtuzumab

- Vacina antipneumocócica conjugada antes de iniciar o tratamento para a LLC;

- SMZ-TMP (800 mg de SMZ 2 x/dia, 3 vezes por semana) se o paciente tiver apresentado $>2$ episódios de infecção grave (necessitando de internação) por ano;

- Considerar o uso de imunoglobulina IV (400 mg/kg a cada 3 semanas) se o paciente apresentou falha à profilaxia com SMZ-TMP (>2 episódios de infecção grave por ano).

b) pacientes que irão receber fludarabina

- SMZ-TMP (800 mg de SMZ 2x/d 3 vezes por semana);

- Isoniazida (300 mg/dia) se o paciente tem história de tuberculose tratada no passado.

c) pacientes que irão receber alemtuzumab

- SMZ-TMP (800 mg de SMZ 2x/d 3 vezes por semana);

- Isoniazida (300 mg/dia) se o paciente tem história de tuberculose tratada no passado;

- Antigenemia para CMV (2x/mês) e ganciclovir $(5 \mathrm{mg} / \mathrm{kg} 12 / 12 \mathrm{~h}$ por 14 dias) se antigenemia positiva. 
- Aciclovir (400 mg VO 3x/dia) se CD4 <50 células $/ \mu \mathrm{L}$, mantendo até $\mathrm{CD} 4>250$ células $/ \mu \mathrm{L}$.

\section{Tratamento}

a) febre em paciente não-neutropênico sem tratamento prévio, ou que tenha recebido quimioterapia com clorambucil ou poliquimioterapia que não contenha fludarabina ou alemtuzumab

- Se paciente não-neutropênico: terapia empírica para bactérias encapsuladas (amoxacilina/clavulanato ou outro ver texto acima);

- Se paciente com neutropenia de baixo risco (ver definição no texto acima) usar uma quinolona respiratória;

- Se paciente com neutropenia de alto risco (ver definição no texto acima) seguir rotina de neutropenia febril da instituição.

b) febre em paciente que esteja em uso de fludarabina ou alemtuzumab

- Antigenemia para CMV é recomendável para o paciente recebendo fludarabina e fundamental para o paciente recebendo alemtuzumab. $\mathrm{O}$ exame deve ser colhido na avaliação inicial de febre de origem obscura, bem como se o paciente não está respondendo a terapia antibiótica de amplo espectro, incluindo pacientes neutropênicos. Colher exames para afastar infecções por micobactérias, $P$. jiroveci, candidíase mucosa e criptococose;

- Iniciar terapia antibiótica empírica visando bactérias encapsuladas, e terapia de amplo espectro se o paciente estiver neutropênico.

c) febre + ulcerações na boca, face ou região perineal em paciente recebendo fludarabina ou alemtuzumab

- Acrescentar aciclovir $\left(250 \mathrm{mg} / \mathrm{m}^{3} 8 / 8 \mathrm{~h}\right.$ ou $400 \mathrm{mg}$ VO $5 x /$ dia).

d) febre + sinais neurológicos em paciente recebendo fludarabina ou alemtuzumab

- Tomografia computadorizada (ou ressonância magnética) de crânio e sorologia para Cryptococcus neoformans e Toxoplasma gondii, exame do líquor, incluindo testes para micobactérias, Listeria e Cryptococcus;

- Iniciar empiricamente SMZ/TMP ou ampicilina + cefepima.

e) Pneumonia em paciente recebendo fludarabina ou alemtuzumab

- Exame do escarro ou lavado broncoalveolar para bactérias, fungos, vírus, micobactérias e $P$. jiroveci;

- Iniciar SMZ-TMP em doses altas;

- Caso não seja disponível a antigenemia para CMV, pacientes que recebem alemtuzumab devem receber ganciclovir $(5 \mathrm{mg} / \mathrm{kg} 12 / 12 \mathrm{~h}$ por 14 dias).

\section{Conclusões}

A LLC é uma neoplasia hematológica que leva a intensa alteração na imunidade humoral e seu tratamento gera defeitos de imunidade mediada por linfócitos T, fagocitária e acentuação da depressão na imunidade humoral. Avaliar o paciente quanto ao estádio de doença, o tipo de terapia em uso e terapias prévias é fundamental para a adequada abordagem de infecção. Entretanto, a despeito do grande impacto negativo das infecções em pacientes com LLC, poucas estratégias preventivas e terapêuticas têm recomendação sólida na literatura. A identificação dos riscos, diagnóstico precoce e tratamento adequado ainda constituem os pilares do tratamento de infecções em pacientes com LLC.

\section{Abstract}

Infection is a frequent complication in patients with chronic lymphocytic leukemia (CLL). The risk of infection is greater with advanced disease and with lower serum immunoglobulin levels. In newly diagnosed patients, the main immunodeficiency is hypogamaglobulinemia, but with different regimens of treatment, other immunodeficiencies occur and contribute to changing the spectrum and increasing the incidence of infection. This paper reviews the current knowledge of the risks and epidemiology of infection according to the different regimens given in the treatment of CLL, and presents recommendations for prophylaxis and treatment of these infections. Such recommendations are rated according to standard scoring schema used in other guidelines to illustrate the strength of the supporting evidence and the quality of the data. Rev. bras. hematol. hemoter. 2005; 27(4):290-300.

Key words: Chronic lymphocytic leukemia; infection; management; guidelines.

\section{Referências Bibliográficas}

1. Morrison VA. Infections in patients with chronic lymphocytic leukemia. In: Cheson BD, editor. Chronic lymphoid Leukemias. Second Edition, revised and expanded. New York: Marcel Dekker, 2001:505-523.

2. Itala $\mathrm{M}$, Helenius $\mathrm{H}$, Nikoskelainen $J$ et al. Infections and serum IgG levels in patients with chronic lymphocytic leukemia. Eur J Haematol 1992;48(5):266-270.

3. Morrison VA. The infectious complications of chronic lymphocytic leukemia. Semin Oncol 1998;25(1):98-106.

4. Molica S, Levato D, Levato L. Infections in chronic lymphocytic leukemia. Analysis of incidence as a function of length of follow-up. Haematologica 1993;78(6):374-377.

5. Robertson TI. Complications and causes of death in B cell chronic lymphocytic leukaemia: a long term study of 105 patients. Aust N Z J Med 1990;20(1):44-50.

6. Rozman C, Montserrat E, Vinolas N. Serum immunoglobulins in B-chronic lymphocytic leukemia. Natural history and prognostic significance. Cancer 1988;61(2):279-283.

7. Griffiths $\mathrm{H}$, Lea J, Bunch $\mathrm{C}$ et al. Predictors of infection in chronic lymphocytic leukaemia (CLL). Clin Exp Immunol 1992;89(3): 374-377. 
8. Tsiodras S, Samonis G, Keating MJ et al. Infection and immunity in chronic lymphocytic leukemia. Mayo Clin Proc 2000;75(10): 1.039-1.054.

9. Heath ME, Cheson BD. Defective complement activity in chronic lymphocytic leukemia. Am J Hematol 1985;19(1):63-73.

10. Schlesinger M, Broman I, Lugassy G. The complement system is defective in chronic lymphatic leukemia patients and in their healthy relatives. Leukemia 1996;10(9):1.509-1.513.

11. Ahmed S, Siddiqui AK, Rossoff L, et al. Pulmonary complications in chronic lymphocytic leukemia. Cancer 2003;98(9):1.912-1.917.

12. Raphael B, Andersen JW, Silber R et al. Comparison of chlorambucil and prednisone versus cyclophosphamide, vincristine, and prednisone as initial treatment for chronic lymphocytic leukemia: long-term follow-up of an Eastern Cooperative Oncology Group randomized clinical trial. J Clin Oncol 1991;9(5):770-776.

13. Molteni A, Nosari A, Montillo M, et al. Multiple lines of chemotherapy are the main risk factor for severe infections in patients with chronic lymphocytic leukemia with febrile episodes. Haematologica 2005; 90(8):1.145-1.147.

13. Wijermans PW, Gerrits WB, Haak HL. Severe immunodeficiency in patients treated with fludarabine monophosphate. Eur J Haematol 1993; 50(5):292-296.

15. Ravandi F, O'Brien S. Infections associated with purine analogs and monoclonal antibodies. Blood Rev 2005;19(5):253-273.

16. Richards S. Fludarabine increases complete response but not survival compared with conventional alkylator-based regimens for previously untreated chronic lymphocytic leukaemia. Cancer Treat Rev 2005; 31(4):332-335.

17. Morrison VA, Rai KR, Peterson BL et al. Impact of therapy With chlorambucil, fludarabine, or fludarabine plus chlorambucil on infections in patients with chronic lymphocytic leukemia: Intergroup Study Cancer and Leukemia Group B 9011. J Clin Oncol 2001;19 (16): 3.611-3.621.

18. Anaissie EJ, Kontoyiannis DP, O'Brien S et al. Infections in patients with chronic lymphocytic leukemia treated with fludarabine. Ann Intern Med 1998;129(7):559-566.

19. Bergmann L, Fenchel K, Jahn B et al. Immunosuppressive effects and clinical response of fludarabine in refractory chronic lymphocytic leukemia. Ann Oncol 1993;4(5):371-375.

20. Leenders A, Sonneveld P, de Marie S. Cryptococcal meningitis following fludarabine treatment for chronic lymphocytic leukemia. Eur J Clin Microbiol Infect Dis 1995; 14(9):826-828.

21. O'Brien S, Kantarjian H, Beran M et al. Results of fludarabine and prednisone therapy in 264 patients with chronic lymphocytic leukemia with multivariate analysis-derived prognostic model for response to treatment. Blood 1993;82(6):1.695-1.700.

22. Silva FA, Matos JO, de Q Mello F, Nucci M. Risk factors for and attributable mortality from tuberculosis in patients with hematologic malignances. Haematologica 2005;90(8):1.110-1.115.

23. Vigouroux S, Morin O, Milpied $\mathrm{N}$ et al. [Cryptococcus neoformans infection in hematologic malignancies]. Rev Med Interne 2000; 21(11): 955-960.

24. Montserrat E, Lopez-Lorenzo JL, Manso F et al. Fludarabine in resistant or relapsing B-cell chronic lymphocytic leukemia: the Spanish Group experience. Leuk Lymphoma 1996;21(5-6):467-472.

25. Keating MJ, O'Brien S, Kontoyiannis D et al. Results of first salvage therapy for patients refractory to a fludarabine regimen in chronic lymphocytic leukemia. Leuk Lymphoma 2002;43(9): 1.755-1.762.

26. Robak T, Blonski JZ, Kasznicki M et al. Cladribine with prednisone versus chlorambucil with prednisone as first-line therapy in chronic lymphocytic leukemia: report of a prospective, randomized, multicenter trial. Blood 2000;96(8):2.723-2.729.

27. Keating MJ, Flinn I, Jain V et al. Therapeutic role of alemtuzumab (Campath-1H) in patients who have failed fludarabine: results of a large international study. Blood 2002;99(10):3.554-3.561.
28. Keating M, Coutre S, Rai K et al. Management guidelines for use of alemtuzumab in B-cell chronic lymphocytic leukemia. Clin Lymphoma 2004;4(4):220-227.

29. Lin TS, Flinn IW, Lucas MS et al. Filgrastim and alemtuzumab (Campath$1 \mathrm{H})$ for refractory chronic lymphocytic leukemia. Leukemia 2005;19(7):1.207-1.210.

30. Wendtner CM, Ritgen M, Schweighofer CD et al. Consolidation with alemtuzumab in patients with chronic lymphocytic leukemia (CLL) in first remission - experience on safety and efficacy within a randomized multicenter phase III trial of the German CLL Study Group (GCLLSG). Leukemia 2004;18(6):1.093-1.101.

31. Cusack JC Jr., Seymour JF, Lerner S et al. Role of splenectomy in chronic lymphocytic leukemia. J Am Coll Surg 1997;185(3):237-243.

32. Horowitz J, Smith JL, Weber TK et al. Postoperative complications after splenectomy for hematologic malignancies. Ann Surg 1996; 223(3):290-296.

33. Gribben JG, Zahrieh D, Stephans K et al. Autologous and allogeneic stem cell transplantation for poor risk chronic lymphocytic leukemia. Blood 2005;106(13):4.389-96.Epub 2005-Aug 30.

34. Dreger P, Brand R, Milligan D et al. Reduced-intensity conditioning lowers treatment-related mortality of allogeneic stem cell transplantation for chronic lymphocytic leukemia: a population-matched analysis. Leukemia 2005;19(6):1.029-1.033.

35. Doney KC, Chauncey T, Appelbaum FR. Allogeneic related donor hematopoietic stem cell transplantation for treatment of chronic lymphocytic leukemia. Bone Marrow Transplant 2002;29(10): 817-823.

36. Dreger P, Michallet M, Schmitz N. Stem-cell transplantation for chronic lymphocytic leukemia: the 1999 perspective. Ann Oncol 2000;11 Suppl 1:49-53.

37. Faderl SJ, Keating MJ. Treatment of chronic lymphocytic leukemia. Curr Hematol Rep 2005;4(1):31-38.

38. Faguet GB. Chronic lymphocytic leukemia: an updated review. J Clin Oncol 1994;12(9):1.974-1.990.

39. Sorror ML, Maris MB, Sandmaier BM et al. Hematopoietic cell transplantation after non-myeloablative conditioning for advanced chronic lymphocytic leukemia. J Clin Oncol 2005;23(16):3.819-3.829.

40. Hughes WT, Rivera GK, Schell MJ et al. Successful intermittent chemoprophylaxis for Pneumocystis carinii pneumonitis. N Engl J Med 1987;316(26):1.627-1.632.

41. Robak T. Alemtuzumab in the treatment of chronic lymphocytic leukemia. BioDrugs 2005;19(1):9-22.

42. Reusser P, Einsele H, Lee J et al. Randomized multicenter trial of foscarnet versus ganciclovir for preemptive therapy of cytomegalovirus infection after allogeneic stem cell transplantation. Blood 2002; 99(4):1.159-1.164.

43. Fraser GA, Walker II. Cytomegalovirus prophylaxis and treatment after hematopoietic stem cell transplantation in Canada: a description of current practices and comparison with Centers for Disease Control/Infectious Diseases Society of America/American Society for Blood and Marrow Transplantation guideline recommendations. Biol Blood Marrow Transplant 2004;10(5): 287-297.

44. Egerer G, Hensel M, Ho AD. Infectious complications in chronic lymphoid malignancy. Curr Treat Options Oncol 2001;2(3):237-244.

45. Agarwal SK, Gupta S, Dash SC et al. Prospective randomised trial of isoniazid prophylaxis in renal transplant recipient. Int Urol Nephrol 2004;36(3):425-431.

46. Carmona L, Gomez-Reino JJ, Rodriguez-Valverde V et al. Effectiveness of recommendations to prevent reactivation of latent tuberculosis infection in patients treated with tumor necrosis factor antagonists. Arthritis Rheum 2005;52(6):1.766-1.772.

47. Grant AD, Charalambous S, Fielding KL et al. Effect of routine isoniazid preventive therapy on tuberculosis incidence among HIV-infected men in South Africa: a novel randomized incremental recruitment study. JAMA 2005;293(22):2.719-2.725. 
48. Mok MY, Lo Y, Chan TM, et al. Tuberculosis in systemic lupus erythematosus in an endemic area and the role of isoniazid prophylaxis during corticosteroid therapy. J Rheumatol 2005; 32(4):609-615.

49. Ravandi F, Anaissie EJ, O'Brien S. Infections in chronic leukemias and other hematological malignancies. In: Wingard JR, Bowden RA, editors. Management of infection in oncology patients. London: Martin Dunitz, 2003:105-128

50. Dykewicz CA. Guidelines for preventing opportunistic infections among hematopoietic stem cell transplant recipients: focus on community respiratory virus infections. Biol Blood Marrow Transplant 2001; 7 Suppl:19S-22S

51. Gafter-Gvili A, Fraser A, Paul M et al. Meta-analysis: antibiotic prophylaxis reduces mortality in neutropenic patients. Ann Intern Med 2005; 142(12 Pt 1):979-995.

52. O'Brien S, Kantarjian H, Beran M et al. Fludarabine and granulocyte colony-stimulating factor (G-CSF) in patients with chronic lymphocytic leukemia. Leukemia 1997;11(10):1.631-1.635.

53. American Society of Clinical Oncology. Recommendations for the use of hematopoietic colony-stimulating factors: evidence-based, clinical practice guidelines. J Clin Oncol 1994;12(11):2.471-2.508.

54. Intravenous immunoglobulin for the prevention of infection in chronic lymphocytic leukemia. A randomized, controlled clinical trial. Cooperative Group for the Study of Immunoglobulin in Chronic Lymphocytic Leukemia. N Engl J Med 1988;319(14): 902-907.

55. Boughton BJ, Jackson N, Lim S et al. Randomized trial of intravenous immunoglobulin prophylaxis for patients with chronic lymphocytic leukaemia and secondary hypogammaglobulinaemia. Clin Lab Haematol 1995;17(1):75-80.

56. Griffiths H, Brennan V, Lea J et al. Crossover study of immunoglobulin replacement therapy in patients with low-grade B-cell tumors. Blood 1989;73(2):366-368.

57. Molica S, Musto P, Chiurazzi F et al. Prophylaxis against infections with low-dose intravenous immunoglobulins (IVIG) in chronic lymphocytic leukemia. Results of a crossover study. Haematologica 1996;81(2):121-126.

58. Gamm H, Huber C, Chapel H et al. Intravenous immune globulin in chronic lymphocytic leukaemia. Clin Exp Immunol 1994;97 Suppl 1:17-20.

59. Sinisalo M, Aittoniemi J, Oivanen Pet al. Response to vaccination against different types of antigens in patients with chronic lymphocytic leukaemia. Br J Haematol 2001;114(1):107-110.

60. Sinisalo M, Aittoniemi J, Kayhty H, Vilpo J. Vaccination against infections in chronic lymphocytic leukemia. Leuk Lymphoma 2003;44(4):649-652.

61. Hughes WT, Armstrong D, Bodey GP et al. 2002 guidelines for the use of antimicrobial agents in neutropenic patients with cancer. Clin Infect Dis 2002;34(6):730-751

62. Fife KH, Barbarash RA, Rudolph T et al. Valaciclovir versus acyclovir in the treatment of first-episode genital herpes infection. Results of an international, multicenter, double-blind, randomized clinical trial. The Valaciclovir International Herpes Simplex Virus Study Group. Sex Transm Dis 1997;24(8):481-486.

63. Meyers JD, Wade JC, Mitchell CD et al. Multicenter collaborative trial of intravenous acyclovir for treatment of mucocutaneous herpes simplex virus infection in the immunocompromised host. Am J Med 1982;73(1A):229-235

64. Shepp DH, Newton BA, Dandliker PS et al. Oral acyclovir therapy for mucocutaneous herpes simplex virus infections in immunocompromised marrow transplant recipients. Ann Intern Med 1985;102(6):783-785.

65. Bertz H, Auner HW, Weissinger F et al. Antimicrobial therapy of febrile complications after high-dose chemo-/radiotherapy and autologous hematopoietic stem cell transplantation--guidelines of the Infectious Diseases Working Party (AGIHO) of the German Society of Hematology and Oncology (DGHO). Ann Hematol 2003; 82 Suppl 2:S167-S174.
66. Einsele H, Bertz H, Beyer J et al. Infectious complications after allogeneic stem cell transplantation: epidemiology and interventional therapy strategies--guidelines of the Infectious Diseases Working Party (AGIHO) of the German Society of Hematology and Oncology (DGHO). Ann Hematol 2003;82 Suppl 2:S175-S185.

67. Ljungman P, Engelhard D, de la CR et al. Vaccination of stem cell transplant recipients: recommendations of the Infectious Diseases Working Party of the EBMT. Bone Marrow Transplant 2005;35 (8):737-746.

68. Nichols WG. Management of infectious complications in the hematopoietic stem cell transplant recipient. J Intensive Care Med 2003;18(6):295-312.

Avaliação: Carlos Sergio Chiattone

(Publicado após acordo do Editor)

Conflito de interesse: Artigo derivado do II Encontro Brasileiro de

Consenso da LLC

Recebido: 11/10/2005

Aceito: 30/10/2005 